\title{
Paracoccidiodomicose em animais silvestres e domésticos
}

\section{Paracoccidioidomycosis in wild and domestic animals}

\author{
Sandra Leite Neves ${ }^{1}$; Tatiane Ferreira Petroni ${ }^{2}$; \\ Paola Fernanda Fedatto ${ }^{2}$; Mario Augusto $\mathrm{Ono}^{3 *}$
}

\section{Resumo}

A paracoccidioidomicose é a micose sistêmica mais prevalente na América Latina, principalmente no Brasil. Apesar de existirem vários estudos sobre o diagnóstico e patologia dessa micose, pouco se conhece sobre a ecologia do agente etiológico Paracoccidioides brasiliensis. Seu habitat ainda não foi determinado, mas provavelmente o fungo vive saprofiticamente no solo. Há também pouca informação quanto a participação de outras espécies de animais na eco-epidemiologia do fungo e os isolamentos obtidos de animais como morcego e pingüim não foram reprodutíveis. Até o presente, o fungo tem sido isolado com maior frequiência do tatu, Dasypus novencinctus. Estudos soroepidemiológicos demonstraram que a infecção é freqüente em cães, principalmente da área rural e recentemente foi relatado o primeiro caso de paracoccidiodomicose natural em cão. O papel de outras espécies de animais na eco-epidemiologia do fungo ainda não está esclarecido

Palavras-chave: Paracoccidioides brasiliensis. Paracoccidiodomicose. Epidemiologia. Animais silvestres. Animais domésticos.

Abstract

Paracoccidiodomycosis is the most prevalent systemic mycosis in Latin América, mainly in Brazil. Although many studies have been done about the pathology and diagnosis of this mycosis little is known about the eco-epidemiology of the etiological agent Paracoccidioides brasiliensis. The fungus habitat also is unknown although is thought that the fungus lives as a saprobe in soil. The participation of other animal species in P. brasiliensis ecology is poorly understood and fungus isolation from animals as bat and penguin were not reproducible. The armadillos Dasypus novencinctus probably play a role in the fungus eco-epidemiology taking into account that several isolates were obtained from these animals. Seroepidemiologic studies have shown that dogs are infected by $P$. brasiliensis mainly in rural areas and recently was reported the first case o natural paracoccidioidomycosis in dogs. The role of other animal species in the eco-epidemiology of $P$. brasiliensis remains doubtful.

Key words: Paracoccidioides brasiliensis. Paracoccidiodomycosis. Epidemiology. Wild animals. Domestic animals.

Bióloga pela Universidade Estadual de Londrina com Especialização em Biologia Aplicada à Saúde, UEL.

Bolsista de Iniciação Científica, Depto. de Ciências Patológicas, Universidade Estadual de Londrina

Docente do Depto. de Ciências Patológicas, UEL. E-mail: marioono@uel.br

* Autor para correspondência 


\section{Introdução}

A paracoccidioidomicose ( $\mathrm{PCM})$ é a micose sistêmica mais importante da América Latina, e os países com maior prevalência são Brasil, Argentina, Colômbia e Venezuela. No Brasil, a maioria dos casos tem sido reportada nas regiões Sudeste, Sul e CentroOeste (BRUMMER; CASTANEDA; RESTREPO, 1993; BLOTTA; CAMARGO, 1993).

O agente etiológico da PCM é o fungo Paracoccidioides brasiliensis, que apresenta dimorfismo termo-dependente. $\mathrm{O}$ fungo cresce a $25^{\circ} \mathrm{C}$ na forma de micélio (forma $\mathrm{M}$ ) enquanto que a $37^{\circ} \mathrm{C}$ e nos tecidos do hospedeiro encontra-se na forma de levedura (forma L) (McEWEN et al., 1987).

A infecção provavelmente ocorre por inalação de propágulos, que posteriormente, nos pulmões, transformam-se em células leveduriformes. Após crescimento no parênquima pulmonar, dependendo do hospedeiro pode disseminar para outros órgãos produzindo uma doença progressiva (McEWEN et al., 1987). A paracoccidioidomicose é mais freqüente no pulmão, fígado, linfonodos, baço, rim, adrenais, mucosas e pele (FRANCO; MONTENEGRO, 1982).

No International Colloquium on Paracoccidioidomycosis realizado em Medellin, Colômbia, 1986, a PCM foi classificada em PCMinfecção, PCM-doença de forma aguda ou subaguda (forma juvenil), forma crônica (tipo adulta) ou forma residual (seqüelas). A PCM crônica pode ser novamente subdividida, em PCM unifocal ou multifocal conforme o número de locais de lesões (FRANCO, 1987).

A PCM-infecção ocorre em indivíduos de ambos os sexos, aparentemente sadios, que residem ou residiram em zona endêmica e que têm reação intradérmica positiva para antígeno de $P$. brasiliensis, porém não há desenvolvimento da doença.

A PCM-doença afeta principalmente trabalhadores rurais do sexo masculino, com idade média de 40 anos. A forma crônica ocorre em mais de $90 \%$ dos pacientes, e é freqüente em indivíduos adultos do sexo masculino. A doença progride lentamente e o período de incubação pode durar meses ou anos. A forma aguda ou juvenil é caracterizada por um período curto de incubação (semanas à meses) e por envolvimento do sistema reticuloendotelial (LONDERO; MELO, 1983).

A PCM não é contagiosa de pessoa a pessoa. A incidência e prevalência dessa micose sistêmica são difíceis de determinar, pois a notificação de sua ocorrência não é compulsória (FERREIRA DA CRUZ; WANKE; GALVÃO- CASTRO, 1987).

O diagnóstico definitivo da PCM pode ser realizado por meio de exame micológico de fluidos broncoalveolares, pus, material de lesões, fluido cerebroespinhal, ou biópsias de tecidos (LACAZ, 1982).

Os ensaios sorológicos também podem auxiliar no diagnóstico da PCM, como imunodifusão em gel, ELISA e Western Blot.

O primeiro antígeno a ser utilizado no imunodiagnóstico da PCM foi o polissacarídeo extraído de $P$. brasiliensis, padronizado por Fava Netto (1961). Diferentes preparações de antígenos variam grandemente na qualidade, dependendo da linhagem do fungo, da fase morfológica, meio de cultura, tempo de inoculação, e técnica desenvolvida (RESTREPO, 1985).

Posteriormente, foi introduzido o exoantígeno, que contém antígenos glicoprotéicos que são reconhecidos pelos soros de pacientes com PCM (BRUMMER et al., 1984; PUCCIA et al., 1986; CAMARGO et al., 1988; MENDES-GIANNINI et al., 1990).

Dentre as diversas glicoproteínas de 16 à 220 kDa produzidas pelo fungo, somente a glicoproteína gp43 é reconhecida por $100 \%$ dos pacientes PCM, sendo considerada o antígeno específico do P. brasiliensis (SOUZA et al., 1997).

Embora tenham sido obtidos grandes avanços em vários aspectos da paracoccidioidomicose, a eco- 
epidemiologia do $P$. brasiliensis ainda não está bem esclarecida. Acredita-se que o habitat do $P$. brasiliensis seja o solo, entretanto, desde o primeiro relato de PCM por Lutz (1908), há poucos isolados a partir dessa fonte. Foram obtidos isolados de amostra de solo da Argentina (NEGRONI, 1966), Venezuela (ALBORNOZ, 1971), e Brasil (SILVA-VERGARA et al., 1998), porém novas tentativas de isolamento não foram bem sucedidas.

Ono et al (2002) observaram que vários agrotóxicos como fungicidas, inseticidas e herbicidas, inibem o crescimento de $P$. brasiliensis "in vitro". Considerando que nas culturas de importância econômica são aplicadas grandes quantidades de agrotóxicos é possível que esse seja um dos fatores que dificultam o isolamento de $P$. brasiliensis a partir de amostras do solo.

Conti-Diaz e Rilla (1989) acreditam que o fungo pode ser encontrado em áreas de reservas florestais, bosques, próximos a rios, tendo como reservatórios naturais anfíbios, peixes e artrópodes.

A dificuldade de isolamento do P. brasiliensis a partir do solo, levou vários pesquisadores à procura de novos mecanismos para auxiliar na busca do habitat desse fungo. Uma das abordagens possíveis seria a utilização de animais sentinela.

\section{Paracoccidioidomicose em cães}

Pereira e Viana (1911) realizaram o primeiro estudo de infecção experimental com $P$. brasiliensis em cão. Os autores inocularam pus de paciente com PCM em um cão e o animal morreu após 22 dias com sinais clínicos de PCM.

Mós, Saliba e Brito (1974), por outro lado, não conseguiram provocar PCM-doença em cães inoculados com suspensão da forma $\mathrm{L}$ de $P$. brasiliensis. Quatorze cães com sorologia negativa para PCM, foram inoculados com P. brasiliensis por via intratesticular. Os animais foram sacrificados para análise histopatológica e tentativa de isolamento do fungo. A resposta imune humoral também foi acompanhada por meio de reação de fixação de complemento e reação de precipitação, porém foi observado positividade apenas na fixação de complemento. Em exame histopatológico do testículo, 24 horas após a inoculação, foi verificado processo inflamatório agudo acompanhado por edema e congestão acentuados. O fungo apresentava-se em número reduzido; porém nenhum dos animais desenvolveu PCM-doença.

Ono et al (2003) realizaram infecção experimental em quatro cães com três meses de idade, com $P$. brasiliensis, por via endovenosa. Todos os animais eram negativos por ELISA e teste intradérmico com gp43 antes da infecção. Uma semana após inoculação, dois animais morreram. A análise histopatológica de pulmões, baço e fígado demonstrou a presença de granulomas com formas características da levedura de $P$. brasiliensis em todos os órgãos, com maior comprometimento dos pulmões. Os autores conseguiram isolar o fungo de amostras de pulmão, fígado e baço dos dois cães que desenvolveram PCM-doença. Em outro estudo de infecção experimental de cães com $P$. brasiliensis, utilizando animais adultos jovens, não foi possível isolar o fungo a partir de amostras de tecidos e nenhum dos animais desenvolveu sinais clínicos de PCM, sugerindo que cães adultos são resistentes ao desenvolvimento da PCM-doença (EISELE et al., 2004).

Mós e Fava Netto (1974), examinaram através da reação de precipitação em tubos e reação de fixação do complemento; soros de 145 cães, sendo 113 procedentes da cidade de São Paulo e 32 de Botucatu. Estes autores observaram positividade de 74,33 e 78,2\% por fixação de complemento em cães de São Paulo e Botucatu respectivamente. Entretanto, não se conseguiu demonstrar a presença de $P$. brasiliensis, quer por exame microscópico direto, quer pelas culturas realizadas a partir de fragmentos de órgãos dos animais sacrificados; também não foram verificadas lesões sugestivas de PCM. 
Recentemente, Ono et al. (2001) realizaram um estudo epidemiológico em cães da região norte do Paraná para detecção de anticorpos anti-gp43 de $P$. brasiliensis por ELISA, e observaram uma positividade de $89,5 \%$ e 48,8\% em cães da área rural e da periferia, respectivamente. Não foi possível isolar P. brasiliensis a partir de amostras de pulmão, fígado e baço de 6 cães com sorologia positiva para PCM.

Fagundes et al. (2002) encontraram uma positividade de $26,5 \%$ em estudo soroepidemiológico com 275 cães de áreas rurais da região de Botucatu (SP), utilizando ELISA com exoantígeno de $P$. brasiliensis.

Ricci et al. (2004) relataram o primeiro caso de PCM-doença em cão. $\mathrm{O}$ animal apresentou aumento dos linfonodos cervicais e a confirmação da doença foi feita por meio de exames histopatológico, de imunohistoquímica e de biologia molecular (PCR) embora não tenha ocorrido isolamento do fungo.

\section{Paracoccidioidomicose em morcegos}

Grose e Tamsitt (1965) capturaram morcegos (Artibeus lituratus) da zona tropical da Colômbia e amostras de material fecal foram coletadas do trato intestinal e semeadas em ágar Sabouraud. Apenas três, de um total de 243 morcegos analisados, apresentaram infecção por $P$. brasiliensis.

Greer e Bolaños (1977) procuraram esclarecer a função dos morcegos na ecologia de $P$. brasiliensis. $\mathrm{Na}$ tentativa de isolar o fungo desses animais, foram cultivados amostras de fezes de 225 morcegos correspondentes a 8 gêneros e 9 espécies. Isolado de $P$. brasiliensis de um paciente com PCMmucocutânea foi preparada e inoculada via oral em 24 animais. Depois do intervalo de 2,4, 6, 8 e 24 horas, os animais foram sacrificados. Esse estudo mostrou a rápida passagem de material ingerido pelo trato digestivo de A. lituratus revelada pela detecção de leveduras viáveis no reto de animais sacrificados 510 minutos após alimentação. Foi observado que células leveduriformes persistiam na área retal até 8 horas, mas elas nunca apareciam em grande número e o fungo morria rapidamente (até 8 horas) no material fecal. Os autores não conseguiram isolar o fungo do trato digestivo de A. lituratus, sugerindo que essa espécie não deve ser importante na disseminação de $P$. brasiliensis na natureza.

\section{Paracoccidioidomicose em eqüinos e bovinos}

Conti-Diaz et al. (1972), no Uruguai, realizaram inquérito epidemiológico de paracoccidiodomicose em 195 cavalos por meio de reação intradérmica com paracoccidioidina. Os autores relataram positividade de $23 \%$. Um dos animais foi reativo também no teste de precipitação embora o soro não tenha apresentado identidade imunológica com soros de pacientes com paracoccidioidomicose.

Gutierrez et al. (1974) realizaram reação intradérmica com antígeno polissacarídico de $P$. brasiliensis em 293 bovinos na Colômbia e relataram positividade em apenas 6 animais. As amostras de soro dos bovinos não apresentaram reatividade nos exames de fixação de complemento e precipitação.

\section{Paracoccidioidomicose em tatus}

O primeiro isolamento de $P$. brasiliensis de tatus foi relatado por Naiff et al. (1986). Os autores inocularam hamsters com amostras do baço e fígado de tatus (Dasypus novemcinctus) capturados no Estado do Pará e isolaram P. brasiliensis de 4 dos 20 tatus estudados. O fungo foi identificado por cultura e exame histopatológico das vísceras dos hamsters. Os tatus com culturas positivas para $P$. brasiliensis eram aparentemente saudáveis. Um dos isolados de tatu foi caracterizado por meio de métodos imunológicos por Vidal et al. (1995). Os autores analisaram o antígeno bruto do isolado por meio de imunodifusão, eletroforese em gel poliacrilamida e immunoblotting e observaram a presença da gp43, principal antígeno de $P$. brasiliensis.

Bagagli et al. (1998) isolaram P. brasiliensis de 
tatus capturados na área endêmica de PCM em Botucatu. Quatro animais foram sacrificados e submetidos à necropsia. Foram obtidos fragmentos do pulmão, baço, fígado e linfonodos mesentéricos e realizado cultura desses órgãos para obtenção da forma L de P. brasiliensis. Os isolados obtidos foram caracterizados por meio de métodos micológicos, imunológicos e de biologia molecular.

Macedo (1999) isolaram P. brasiliensis de tatus D. novemcinctus em Goiás. Outras espécies de tatu foram estudadas (Euphractus sexcintus e D. septemcinctus), porém não foi possível isolar o fungo a partir das mesmas. Novos isolados do fungo foram obtidos de tatus capturados em Ibiá, Minas Gerais (SILVA-VERGARA et al., 2000).

Foram obtidos também isolados de $P$. brasiliensis a partir de tatus das espécies $D$. novemcinctus (CORREDOR et al., 1999) e Cabassous centralis (CORREDOR et al., 2005) em áreas endêmicas para PCM na Colômbia.

Os vários isolados de $P$. brasiliensis obtidos de tatus $D$. novemcinctus capturados em áreas endêmicas de PCM e a demonstração de que esses animais podem apresentar PCM-doença (SILVAVERGARA; MARTINEZ, 1999) confirmam o papel desse animal na eco-epidemiologia do fungo.

\section{Paracoccidioidomicose em primatas}

Há apenas um relato de PCM-doença em um macaco proveniente da Bolívia, que apresentava lesões macroscópicas no trato gatrointestinal e fígado e uma grande massa nodular firme e branca na superfície interna e externa do fígado estendendo-se ao lóbulo anterior médio. O exame histopatológico mostrou a presença de uma ulcera gástrica que havia penetrado a mucosa e músculos e presença de $P$. brasiliensis, porém não foi feito o isolamento do fungo (JOHNSON; LANG, 1977).

Costa et al. (1995) avaliaram, por meio de teste intradérmico com paracoccidioidina, 49 primatas mantidos em cativeiro, das espécies Cebus apella $(\mathrm{n}=33)$ e Callithrix jacchus $(\mathrm{n}=16)$ e observaram positividade de $22,4 \%$.

\section{Paracoccidioidomicose em gambás}

Silva-Vergara et al (2001) tentaram isolar $P$. brasiliensis de gambás (Didelphis albinventris) na região do Triângulo Mineiro, Estado de Minas Gerais, Brasil. Vinte animais D. albiventris foram sacrificados e amostras do pulmão, fígado e baço foram semeados em meio Fava Netto. O isolamento também foi tentado por meio de inoculação de camundongos, por via intraperitonial, com as amostras homogeneizadas, porém não foi observado nenhum crescimento de colônia sugestiva de $P$. brasiliensis.

O exame histopatológico de vísceras de $D$. albiventris não demonstrou nenhuma lesão granulomatosa ou estrutura similar ao fungo, sugerindo que gambás não são freqüentemente infectados pelo fungo ou ainda que esses animais são resistentes ao desenvolvimento da PCM-doença.

\section{Paracoccidioidomicose em pingüim}

Em 1989, Gezuele surpreendentemente isolou um fungo com características de $P$. brasiliensis em fezes de um pingüim (Pygoscelis adeliae) da Antártida. Esse isolado foi posteriormente caracterizado por Garcia et al. (1993) por meio de métodos micológicos e imunológicos. A amostra pingüim (forma $\mathrm{M}$ ) foi cultivada e inoculada em cobaios por via intratesticular. Após 15 dias de inoculação, os animais foram sacrificados e o testículo apresentava abscessos, com reação granulomatosa. O isolado também produziu gp43, o principal antígeno de $P$. brasiliensis, que foi demonstrada por meio de eletroforese em gel poliacrilamida, imunodifusão e imunoeletroforese (GARCIA et al., 1993).

O significado desse isolamento permanece indefinido; pois o é pouco provável que o fungo possa sobreviver no ambiente da Antártida. 


\section{Considerações Finais}

Entre os vários relatos de PCM em animais, observa-se uma falta de reprodutibilidade nos estudos, exceto em tatus, que têm permitido o isolamento de $P$. brasiliensis a partir de diferentes estados brasileiros e também da Colômbia. Muitas características fazem do gênero Dasypus, animais importantes para pesquisa de seu papel na ecoepidemiologia do P. brasiliensis. A capacidade de escavar o solo a grandes profundidades, provavelmente expõe estes animais com maior facilidade ao provável habitat desse fungo. Adicionalmente, seus hábitos alimentares de destruição de colônias de formigas, coleta de folhas podem facilitar e aumentar essa exposição. A distribuição desses animais coincidem com as áreas endêmicas de PCM. Fatores ecológicos como florestas, abundância em cursos de rios, estação curta de inverno, verão chuvoso e temperaturas de 14 e $27^{\circ} \mathrm{C}$ favorecem o estabelecimento desses animais e sua possível infecção natural.

Os cães, aparentemente, também possuem um papel na eco-epidemiologia do P. brasiliensis, considerando os altos índices de infecção observados em cães de áreas endêmicas de PCM e o recente relato de PCM-doença nesta espécie. É de extrema importância o prosseguimento dos estudos sobre PCM em cães pois casos dessa importante micose sistêmica podem estar ocorrendo nas áreas endêmicas e não estão sendo devidamente diagnosticadas pelos veterinários. Em relação à $\mathrm{PCM}$ em cães é importante também a utilização desses animais como marcadores epidemiológicos, que poderia auxiliar na busca do habitat do fungo.

A importância de outros animais domésticos ou silvestres ainda necessita de comprovação. Dado o grande número de espécies animais que estão em risco de infecção por $P$. brasiliensis em áreas endêmicas é provável que num futuro próximo a PCM seja descrita em outras espécies de animais.

O enigma do verdadeiro habitat do $P$. brasiliensis deve ser elucidado através de um esforço conjunto de pesquisadores de diferentes áreas que pela diversidade de técnicas e conhecimentos, consigam finalmente responder a essa importante pergunta.

\section{Agradecimentos}

Os autores agradecem ao CNPq, CAPES, Fundação Araucária e PROPPG/UEL pela concessão de auxílio financeiro para a realização deste trabalho.

\section{Referências}

ALBORNOZ, M. B. Isolation of Paracoccidioides brasiliensis from rural soil in Venezuela. Sabouraudia, Oxfordshire, Inglaterra v.9, p.248-253, 1971.

BAGAGLI, E. Isolation of Paracoccidioides brasiliensis from armadillos (Dasypus novemcinctus) captured in an area of paracoccidioidomycosis. American Journal of Tropical Medicine and Hygiene, Mclean, v.58, p.505-512, 1998.

BLOTTA, M. H. S. L.; CAMARGO, Z. P. Immunological response to cell-free antigens of Paracoccidioides brasiliensis: relationship with clinical forms of paracoccidioidomycosis. Journal of Clinical Microbiology, Washington, v.31, p.671-676, 1993.

BRUMMER, E.; CASTANEDA, E.; RESTREPO, A. Paracoccidioidomycosis: An update. Clinical Microbiology Reviews, Washington, v.6, n.2, p.89-117, 1993.

BRUMMER, E.; RESTREPO, A.; STEVENS, D. A.; AZZI, R.; GOMEZ, A.; HOYOS, G.; McEWEN, J.; CANO, L.; DeBEDOUT, C. Murine model of paracoccidioidomycosis. Producion of fatal acute pulmonary or chronic pulmonary and disseminated disease: immunological and pathological observations. Journal of Experimental Pathology, Oxford, v.1, p.241, 1984.

CAMARGO, Z. P.; UNTERKIRCHER, C. S.; CAMPOY, S. P.; TRAVASSOS, L. R. Production of Paracoccidioides brasiliensis exoantigens for immunodiffusion tests. Journal of Clinical Microbiology, Washington, v.26, p.2147-51, 1988.

CONTI-DIAZ, I. A.; ALVAREZ, B. J.; GEZUELE, E.; GONZALEZ MARINI, H.; DUARTE, J.; FALCON., J. Intradermal reaction survey with paracoccidioidin and histoplasmin in horses. Revista Do Instituto de Medicina Tropical De Sao Paulo, São Paulo, v.14, p.372-376, 1972. 
CONTI-DIAZ, I. A.; RILLA, F. D. Hypoteses sobre el nicho ecologico de Paracoccidioides brasiliensis. Revista Medica Del Uruguay, Montevideo, v.5, p.97-103, 1989.

CORREDOR, G. G.; CASTA-O, J.H.; PERALTA, L. A.; D’EZ, S.; ARANGO, M.; McEWEN, J.; RESTREPO, A. Isolation of Paracoccidioides brasiliensis from the nine-banded armadillo Dasypus novemcinctus, in na endemic area for paracoccidioidomycosis in Colombia. Revista Iberoamericana De Micologia, Barcelona, v.16, p.216220, 1999.

CORREDOR, G. G.; PERALTA, L. A.; CASTANO, J. H.; ZULUAGA, J. S.; HENAO, B.; ARANGO, M.; TABARES, A. M.; MATUTE, D. R.; McEWEN, J. G.; RESTREPO, A. The naked-tailed armadillo Cabassous centralis (Miller 1899): a new host to Paracoccidioides brasiliensis. Molecular identification of the isolate. Medical Mycology, Oxford, v.43, p.275-280, 2005.

COSTA, E. O.; DINIZ, L. S.; FAVA NETTO, C.; ARRUDA, C.; DAGLI, M. L. Delayed hypersensitivity test with paracoccidioidin in captive Latin American wild mammals. Journal of Medical and Veterinary Mycology, Oxfordshire, v.33, p.39-42, 1995.

EISELE,R.C:;JULIANI,L.C.;BELITARDO,D.R.;TANO,E.N.; ESTEVÃO,D.; BRACARENSE, A.P.F.L.; CAMARGO,Z.P.; $\mathrm{ONO}, \mathrm{M}$. A. Immune response in dogs experimentally infected with Paracoccidioides brasiliensis. Medical Mycology; Oxford, v.42,p.549-53,2004.

FAGUNDES, R. Q.; ARAÚJO JÚNIOR., J. P.; MODOLO, R.; BAGAGLI, E. Serological detection of Paracoccidioidomycosis in dogs from the endemic area of Botucatu-SP, Brazil. Pirenópolis-GO: ARBS, 2002.

FAVA NETTO, C. Contribuição para o estudo imunológico de blastomicose de Lutz. Revista do Instituto Adolpho Lutz, São Paulo, v.21, p.99-194, 1961.

FERREIRA-DA-CRUZ, M. F.; WANKE, B.; GALVÃOCASTRO, B. Prevalence of paracoccidioidomycosis in hospitalized adults in Rio de Janeiro. Mycopathologia, Den Haag, Holanda, v.97, p.61-64, 1987.

FRANCO, M. Paracoccidioidomycosis: a recently proposed classification on its clinical forms. Revista da Sociedade Brasileira de Medicina Tropical, Rio De Janeiro, v.20, p.129-133, 1987.

FRANCO, M.; MONTENEGRO, M. R. G. Anatomia pathologica. In: DEL NEGRO, G.; LACAZ, C. S.; FIORILLO, A. M. (Ed.). Paracoccidiodomicose: Blastomicose Sulamericana. São Paulo: Sarvier, 1982. p.97-117.

GARCIA, N. M.; DEL NEGRO, G. M.B; HEINS-VACCARI, E. M.; MELO, N. T.; ASSIS, C. M.; LACAZ, C. S. Paracoccidioides brasiliensis: a new strain isolated from a fecal matter of a penguin. Revista do Instituto de Medicina Tropical de Sao Paulo, São Paulo, v.35, p.227235, 1993.
GREER, D. L.; BOLANÕS, B. Role of Bats in the ecology of Paracoccidioides brasiliensis in the intestinal tract of frugivorous bat, Artibeus lituratus. Sabouraudia, Oxfordshire, v.15, p.273-283, 1977.

GROSE, E.; TAMSITT, J. R. Paracoccidioides brasiliensis recovered from the intestinal tract of three bats (Artibeus Literatus) in Colômbia. Sabouraudia, Oxfordshire, v.4, p.124-125, 1965.

GUTIERREZ, A. H.; CEBALLOS, G.; FERRER, H. I.; RANGEL, O. Encuesta sobre tuberculosis, histoplasmosis y paracoccidioidomicosis en ganado lechero del Valle del Aburra. Antioquia Medica, Medellin, Colombia, v.24, p.339-358, 1974.

JOHNSON, W. D.; LANG, G. M. Paracoccidioidomycosis (South American Blastomycosis) in a Squirel Monkey (Saimiri sciureus). Veterinary Pathology, Washington, v.14, p.368-371, 1977.

LACAZ, C. S. Aspectos clínicos gerais. Formas polares de paracoccidiodiomicose. In: Del NEGRO, G.; LACAZ, C. S.; FIALHO, A. M. Paracoccidioidomycosis. São Paulo: Sarvier - EDUSP, 1982.

LONDERO, A. T.; MELLO, I. S. Paracoccidioidomycosis in childhood. A critical review. Mycopathologia, Den Haag, Holanda, v.82, p.49-55, 1983.

LUTZ, A. Uma micose pseudo-coccidica localizada na boca e observada no Brasil: contribuição ao conhecimento das hypho-blastomycoses americanas. Brasil - Medico, Rio de Janeiro, v.22, p.121, 1908.

MACEDO, R. C. L. Infecção natural de tatus por Paracoccidioides brasiliensis em Serra da Mesa, Goiás: Estudo preliminar. CONGRESSO BRASILEIRO DE MICOLOGIA, 2., 1999, Rio de Janeiro. Anais... Rio de Janeiro, 1999. p.192.

McEWEN, J. G.; BEDOYA, V.; PATIÑO, M. M.; SALAZAR, M. E.; RESTREPO, A. Experimental murine paracoccidioidomycosis induced by inhalation of conidia. Journal of Medical and Veterinary Mycology, Oxfordshire, v.25, p.165-175, 1987.

MENDES-GIANNINI, M. J. S.; BUENO, J. P.; SHIKANAIYASUDA, M. A.; STOLF, A. M.; MASUDA, A.; AMATO NETO, V.; FERREIRA, A. W. Antibody response to 43KDa glycoprotein of Paracoccidioides brasiliensis as a marker for the evaluation of patients under treatment. American Journal of Tropical Medicine and Hygiene, Mclean, v.43, n.2, p.200-206, 1990.

MÓS, E. N.; FAVA NETTO, C. Contribuição ao estudo da paracoccidiodomicose. I. Possível papel epidemiológico dos cães. Estudo sorológico e anatomo-patológico. Revista do Instituto de Medicina Tropical de Sao Paulo, São Paulo, v.16, p.154-159, 1974. 
MÓS, E. N.; SALIBA, A. M.; BRITO, T. Contribuição ao estudo da paracoccidiodomicose. II. Infecção experimental do cão. Revista do Instituto de Medicina Tropical de Sao Paulo, São Paulo, v.16:, p.232-237, 1974.

NAIFF, R. D.; FERREIRA, L. C. L.; BARRET, T. V.; NAIF, M. F.; ARIAS, J. R. Paracoccidioidomicose enzoótica em tatus (Dasypus novemcinctus) no estado do Pará. Revista do Instituto de Medicina Tropical de Sao Paulo, São Paulo, v.28, p.19-27, 1986.

NEGRONI, P. El Paracoccidioides brasiliensis vive saprofiticamente en el suelo argentino. Prensa Medica Argentina, Buenos Aires, v.53, p.2831-2832, 1966.

ONO, M. A.; ITANO, E. N.; MIZUNO, E. H.; CAMARGO, Z. P. Inhibition of Paracoccidioides brasiliensis by pesticides: Is this a partial explanation for the difficulty in isolating this fungus from the soil? Medical Mycology, Oxford, v.40, p.493-499, 2002.

ONO, M. A.; KISHIMA, M. O.; ITANO, E. N.; BRACARENSE, A. P. F. R. L; CAMARGO, Z. P. Experimental paracoccidioidomycosis in dogs. Medical Mycology; Oxford., v.41, n.3, p.265-268, 2003.

ONO, M.A.; BRACARENSE, A. P. F. R. L.; MORAIS, H. A.; TRAPP, S. M.; BELITARDO, D. R.; CAMARGO, Z. P. Canine paracoccidioidomycosis: A seroepidemiologic study. Medical Mycology, Oxford, v.39, p.277-282, 2001.

PEREIRA, M.; VIANNA, G. A propósito de um caso de blastomicose (Pyohemia blastomycotica), Arquivo Brasileiro de Medicina Veterinária e Zootecnia, Belo Horizonte, v.1, p.65-83, 1911.

PUCCIA, R.; SCHENKMAN, S.; GORIN, P.A.J.; TRAVASSOS, L.R. Exocellular components of Paracoccidioides brasiliensis: identification of a specific antigen. Infecton Immunology, v. 53, p. 199-206, 1986.

RESTREPO, A. The ecology of Paracoccidioides brasiliensis a puzzle still unsolved. Sabouraudia, v. 23, p.323-34, 1985.
RICCI, G.; MOTA, F.T.; WAKAMATSU, A.; SERAFIM, R.C.; FRANCO M. Canine paracoccidioidomycosis. Medical Mycology, Oxford, v. 42, p. 379-383, 2004.

SILVA-VERGARA, M. L. et al. The marsupial Didelphis albiventris is an improbable host of Paracoccidioides brasiliensis in na endemic area of paracoccidioidomycosis in Minas Gerais, Brazil. Memórias do Instituto Oswaldo Cruz, Rio de Janeiro, v. 96, p. 771-772, 2001.

SILVA-VERGARA, M.L.; MARTINEZ, R. Role of the armadillo Dasypus novemcinctus in the epidemiology of paracoccidioidomycosis. Mycopathologia, Den Haag, v. 144, p. 131-133, 1999.

SILVA-VERGARA, M.L.; MARTINEZ, R.; CAMARGO, Z.P.; MALTA, M.H.; MAFFEI, C. M.; CHADU, J.B. Isolation of Paracoccidioides brasiliensis from armadillos (Dasypus novemcinctus) in an area where the fungus was recently isolated from soil. Medical Mycology, Oxford, v. 38, p. 193-199, 2000.

SILVA-VERGARA, M.L.; MARTINEZ, R.; CHADU, A.; MADEIRA, M.; FREITAS-SILVA, G.; LEITE MAFFEI, C.M. Isolation of a Paracoccidioides brasiliensis strain from the soil of a coffee plantation in Ibiá, State of Minas Gerais, Brazil. Medical Mycology, Oxford, v. 36, p. 37-42, 1998.

SOUZA, M. C.; GESZTESI, J.L.; SOUZA, A.R.; MORAES, J.Z.; LOPES, J.D.; CAMARGO, Z.P. Differences in reactivity of paracoccidioidomycosis sera with gp43 isoforms. Journal of Medical and Veterinary Mycology, Oxfordshire v.35, p.13-18, 1997.

VIDAL, M.S.M.; MELO, N.T.; GARCIA, N.M.; DEL NEGRO, G.M.; ASSIS, C.M.; HEINS-VACCARI, E.M.; NAIFF, R.D.; MENDES, R.P.; LACAZ, C.S. Paracoccidioides brasiliensis. a mycologic and immunochemical study of a sample isolated from an armadillo (Dasypus novemcinctus). Revista do Instituto de Medicina Tropical de São Paulo, São Paulo, v.37, n.1, p. 43-49, 1995. 